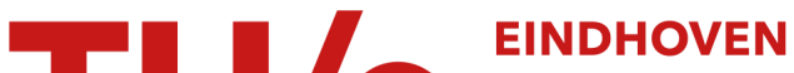 \\ UNIVERSITY OF \\ TECHNOLOGY
}

\section{Spin-up in a rectangular tank with discontinious topography}

\section{Citation for published version (APA):}

van de Konijnenberg, J. A., \& Heijst, van, G. J. F. (1996). Spin-up in a rectangular tank with discontinious topography. Physics of Fluids, 8(11), 2943-2952. https://doi.org/10.1063/1.869073

DOI:

10.1063/1.869073

Document status and date:

Published: 01/01/1996

\section{Document Version:}

Publisher's PDF, also known as Version of Record (includes final page, issue and volume numbers)

\section{Please check the document version of this publication:}

- A submitted manuscript is the version of the article upon submission and before peer-review. There can be important differences between the submitted version and the official published version of record. People interested in the research are advised to contact the author for the final version of the publication, or visit the $\mathrm{DOI}$ to the publisher's website.

- The final author version and the galley proof are versions of the publication after peer review.

- The final published version features the final layout of the paper including the volume, issue and page numbers.

Link to publication

\section{General rights}

Copyright and moral rights for the publications made accessible in the public portal are retained by the authors and/or other copyright owners and it is a condition of accessing publications that users recognise and abide by the legal requirements associated with these rights.

- Users may download and print one copy of any publication from the public portal for the purpose of private study or research.

- You may not further distribute the material or use it for any profit-making activity or commercial gain

- You may freely distribute the URL identifying the publication in the public portal.

If the publication is distributed under the terms of Article 25fa of the Dutch Copyright Act, indicated by the "Taverne" license above, please follow below link for the End User Agreement:

www.tue.nl/taverne

Take down policy

If you believe that this document breaches copyright please contact us at:

openaccess@tue.nl

providing details and we will investigate your claim. 


\title{
Spin-up in a rectangular tank with a discontinuous topography
}

\author{
J. A. van de Konijnenberg and G. J. F. van Heijst \\ Fluid Dynamics Laboratory, Department of Technical Physics, Eindhoven University of Technology, \\ P.O. Box 513, 5600 MB Eindhoven, The Netherlands
}

(Received 20 March 1996; accepted 19 July 1996)

\begin{abstract}
The spin-up from rest to a state of solid-body rotation in a tank with one or more stepwise changes in depth is studied experimentally. Since flow across the steps is counteracted by the rotation acquired by the fluid in the course of the spin-up process, eventually the flow is forced into a cellular pattern determined by the position of the steps. However, if the initial flow field has little resemblance with the quasisteady pattern imposed by the topography, most of the energy of the flow may be dissipated before the organization into the preferred pattern is complete. (C) 1996 American Institute of Physics. [S1070-6631(96)01911-3]
\end{abstract}

\section{INTRODUCTION}

Two-dimensional flows have long been known for their unique properties, which give them a special place in fluid dynamics. Whereas in three-dimensional flows, large-scale structures tend to break up into ever smaller ones, twodimensional flows show a tendency to merge small vortices into well-defined structures with relatively long lifetimes. This phenomenon has been observed in both experiments ${ }^{1}$ and numerical simulations, ${ }^{2,3}$ and is often referred to as selforganization. The formation and persistence of large twodimensional vortex structures is particularly relevant for atmospheric and oceanic flows, since the relative thinness of the atmosphere and the oceans causes these large-scale flows to be essentially two dimensional.

Two-dimensional flows are generally unstable to threedimensional perturbations, and require geometrical constraints or an external forcing to support their existence. Such forcing may arise from a stratified of rotating environment or from a magnetic field. In this paper, we study the formation of vortex patterns in experiments in which the flow is stabilized by its rotation. These experiments consist of a sudden increase in the angular velocity of a tank filled with water. The resulting flow relative to the tank walls gradually adapts itself to the higher angular velocity in a process called spin-up. The background rotation of the flow is slowly established in the course of the experiment, and may be insufficient to stabilize the flow in the beginning of the experiment. However, as the relative flow decays, the rotation of the flow suppresses vertical motions more strongly, and the flow becomes approximately two dimensional.

Early investigations on spin-up concentrated on the azimuthally symmetric flow in a circular tank or between two parallel plates of infinite extent. ${ }^{4,5}$ In that case, the flow preserves its circular symmetry, and slowly spins up due to a weak secondary flow driven by Ekman layers at the bottom and top boundaries of the fluid. In later investigations, spin-up geometries with noncircular cross section were considered. ${ }^{6-8}$ Unless the flow is highly viscous or the deviation from a circular shape is very small, the flow will separate from the sidewall. The transport of vorticity from the sidewall boundaries into the interior of the flow leads to the formation of vortices, so that the streamline pattern loses its resemblance to the shape of the domain.

Most earlier experiments in noncircular geometries were performed in a tank with a flat bottom. In that case, the flow may as a first approximation be treated as two dimensional, with a small divergence caused by the pumping of the Ekman layers. As the kinematic viscosity is assumed to be small, and the relative flow eventually becomes very weak, the flow becomes approximately geostrophic (see Sec. II). In the absence of bottom topography, there is no predetermined restriction on the appearance of the quasisteady flow, which is said to be geostrophically free. In such experiments, the flow usually evolves into a pattern of large-scale vortices that may exist for many eddy turnover times. In a tank with a bottom topography, however, the quasisteady flow is restricted to following the isolines of the topography. If the topography is such that there are no closed topographic contours, as is the case in a tank with a uniform slope, this implies that no geostrophic flow is possible. Such experiments were performed by Van Heijst et al., ${ }^{9}$ who studied spin-up in a rectangular tank with a uniformly sloping bottom. In that case, the flow remains unsteady during the entire experiment.

In this paper, we consider the spin-up in a tank with a bottom topography consisting of piecewise flat sections. By this choice, the presence of a bottom topography is combined with the possibility for geostrophic flow within a number of subdomains of the geometry. In the geometries chosen in this paper, the flow immediately after the start of the experiment is almost perpendicular to the topographic step, so one may expect a transition to a geostrophic state in accordance with the restrictions imposed by the topography. Experiments have been performed in four geometries, each consisting of a small number of shallow and deep square regions of $40 \times 40$ $\mathrm{cm}$. These geometries are idealized in the sense that each square can harbor a circular vortex, so that self-organization effects become more pronounced. These four geometries are represented in Fig. 1.

In Sec. II, a brief outline of geostrophic flows, the spin-up through Ekman pumping and the calculation of the initial flow is given. The experimental setup is described in Sec. III. In Sec. IV, experimental results for stream function 

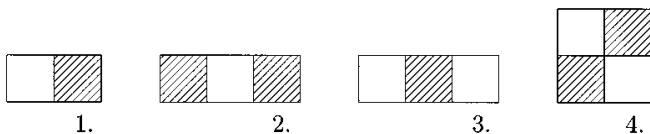

FIG. 1. Geometries studied in this paper. Each square has sides of $40 \mathrm{~cm}$. The shaded regions are $15 \mathrm{~cm}$ deep, the unshaded regions are $20 \mathrm{~cm}$ deep.

and vorticity are presented for all four geometries. Finally, some general conclusions are drawn in Sec. V.

\section{THEORY}

\section{A. Geostrophic flows}

As in this paper the motion of water relative to a tank rotating with constant angular velocity $\boldsymbol{\Omega}$ is the main interest, we consider the incompressible Navier-Stokes equations, given in a reference frame corotating with the tank by

$$
\frac{\partial \mathbf{u}}{\partial t}+(\mathbf{u} \cdot \nabla) \mathbf{u}=-\frac{1}{\rho} \nabla p_{\text {red }}-2 \boldsymbol{\Omega} \times \mathbf{u}+\nu \nabla^{2} \mathbf{u} .
$$

In this equation, $\mathbf{u}$ is the velocity, $t$ the time, $\nu$ the kinematic viscosity, and $p_{\text {red }}$ the reduced pressure, given by

$$
\frac{p_{\text {red }}}{\rho}=\frac{p}{\rho}+g z-\frac{1}{2} \Omega^{2} r^{2},
$$

with $\rho$ the density, $\mathbf{g}$ the acceleration caused by gravity and $r$ the distance from the rotation axis. If the nonlinear and viscous terms are negligible and if the flow is stationary, there is an equilibrium between the gradient of the reduced pressure and the Coriolis force:

$$
\mathbf{0}=-\frac{1}{\rho} \nabla p_{\text {red }}-2 \boldsymbol{\Omega} \times \mathbf{u} .
$$

Flows obeying (3) are called geostrophic.

This limiting case is relevant for spin-up theory, since if the Rossby number $U / \Omega L$ is small, (3) describes to lowest order the flow in the interior, that is, outside the viscous boundary layers at the walls. ${ }^{10}$ Moreover, the equation has special relevance for geophysical fluid dynamics, since in many cases the large scale motions in the oceans and the atmosphere are approximately geostrophic. ${ }^{11}$ After taking the curl of (3) and applying a vector identity, one finds that

$$
(\boldsymbol{\Omega} \cdot \nabla) \mathbf{u}=\mathbf{0} .
$$

This equation is known as the Taylor-Proudman theorem; it states that the velocity field in every plane perpendicular to $\boldsymbol{\Omega}$ is the same. In a laboratory experiment using a tank rotating around a vertical axis, the Taylor-Proudman theorem implies that in the geostrophic limit, there is no velocity component in the vertical direction, and the velocity field is the same at every height.

This has particularly remarkable consequences for a tank with a bottom topography, since in that case the topography dictates the appearance of the entire flow field. Due to the condition of zero normal velocity at the surface, the vertical velocity vanishes identically throughout the domain. Thus geostrophic flows are strictly horizontal, and therefore fluid particles are unable to cross isolines of topography. In this respect there is a fundamental difference between certain types of geometries. If the isolines of the topography are closed curves, such as in the case of a conical hill, streamlines must coincide with them. Such flows are called geostrophically guided. If topography isolines intersect the sidewalls, such as in a tank with a uniformly sloping bottom, no geostrophic flow is possible. Such flows are called geostrophically blocked. In regions where the bottom is flat, the flow may assume any streamline pattern; such flows are called geostrophically free.

\section{B. Ekman pumping: The spin-up time scale}

In a geometry with a flat bottom, it is convenient to use the two-dimensional vorticity equation, derived from the curl of (1):

$$
\frac{\partial \omega}{\partial t}+u \frac{\partial \omega}{\partial x}+v \frac{\partial \omega}{\partial y}=-(2 \Omega+\omega)\left(\frac{\partial u}{\partial x}+\frac{\partial v}{\partial y}\right)+\nu \nabla^{2} \omega .
$$

Although the three-dimensional flow is assumed to be strictly incompressible, the vorticity equation is given here in its compressible form. The reason is that $\partial u / \partial x+\partial v / \partial y$ will be slightly nonzero because of a small vertical velocity component at the bottom of the fluid, provided by the so-called Ekman boundary layer. It can be shown ${ }^{4,8,10}$ that if the relative velocities are small (that is, if the Rossby number $U / \Omega L$ is small), the two-dimensional divergence is given by

$$
\frac{\partial u}{\partial x}+\frac{\partial v}{\partial y}=\frac{1}{2} \sqrt{E} \omega
$$

with $E=\nu / \Omega H^{2}$ the Ekman number; $H$ denotes the distance from the fluid to the free surface, or, if the fluid is covered with a rigid lid, half the distance from the bottom to this rigid lid. Inserting (6) in (5), we find

$$
\frac{\partial \omega}{\partial t}+u \frac{\partial \omega}{\partial x}+v \frac{\partial \omega}{\partial y}=-\frac{1}{2} \sqrt{E}(2 \Omega+\omega) \omega+\nu \nabla^{2} \omega .
$$

The linear Ekman pumping condition (6) is sometimes also used if the Rossby number is not small; in that case the nonlinear vorticity equation (7) should be used. If the Rossby number is small, $2 \Omega+\omega$ may be replaced by $2 \Omega$ and the advective term can be neglected, so that (7) reduces to

$$
\frac{\partial \omega}{\partial t}=-\Omega \sqrt{E} \omega+\nu \nabla^{2} \omega .
$$

If the viscous term is small, this leads to an exponential decay on a time scale

$$
\tau=\frac{H}{\sqrt{\nu \Omega}} .
$$

\section{Starting flow}

The flow immediately after the onset of the rotation is referred to as the starting flow. Since the absolute vorticity in the nonrotating system is unaffected by the sudden motion of the sidewalls, the relative vorticity of the starting flow has a uniform value $-2 \Omega$. In a rectangular tank $-L<x<L,-B$ 

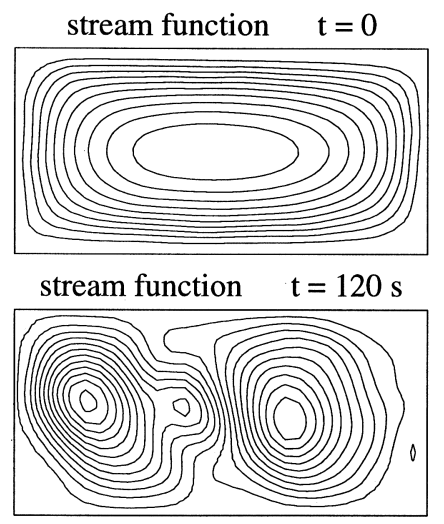

vorticity $t=0$

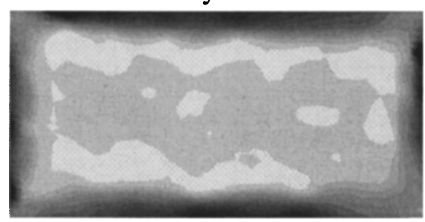

vorticity $t=120 \mathrm{~s}$
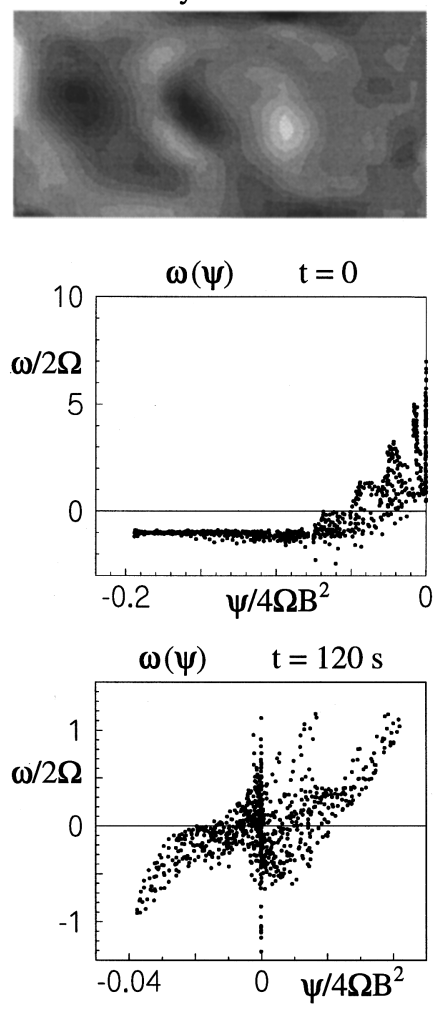

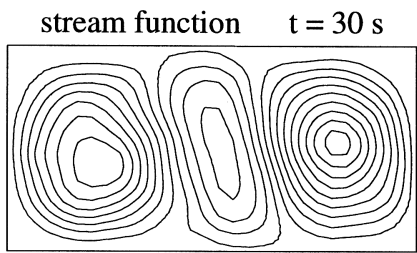

stream function $\mathrm{t}=240 \mathrm{~s}$

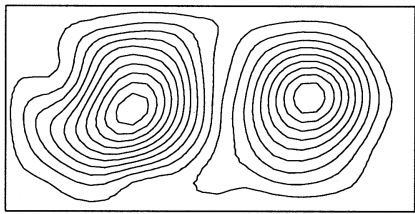

vorticity $t=30 \mathrm{~s}$

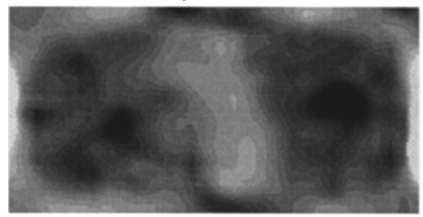

vorticity $t=240 \mathrm{~s}$
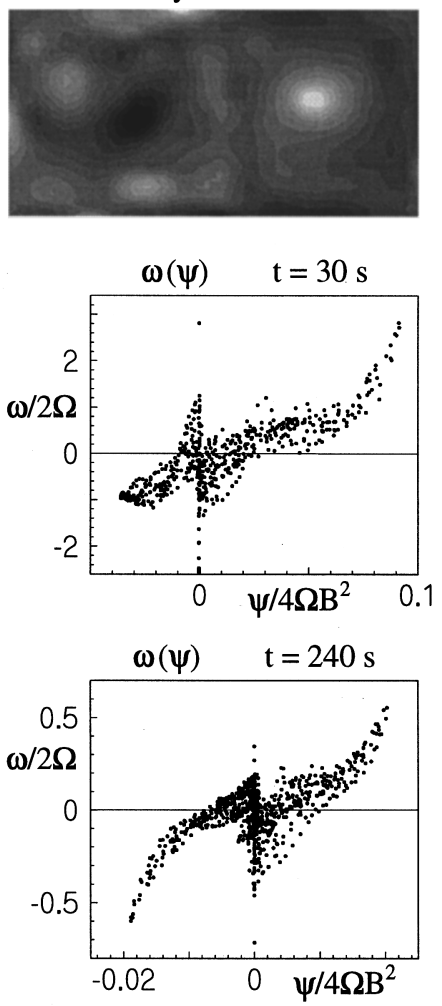

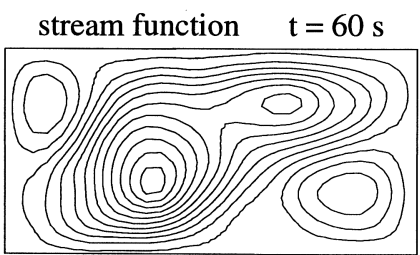

stream function $\mathrm{t}=480 \mathrm{~s}$

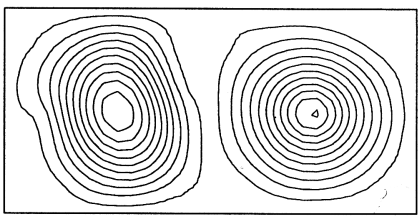

vorticity $t=60 s$

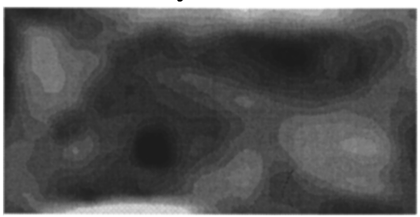

vorticity $t=480 \mathrm{~s}$
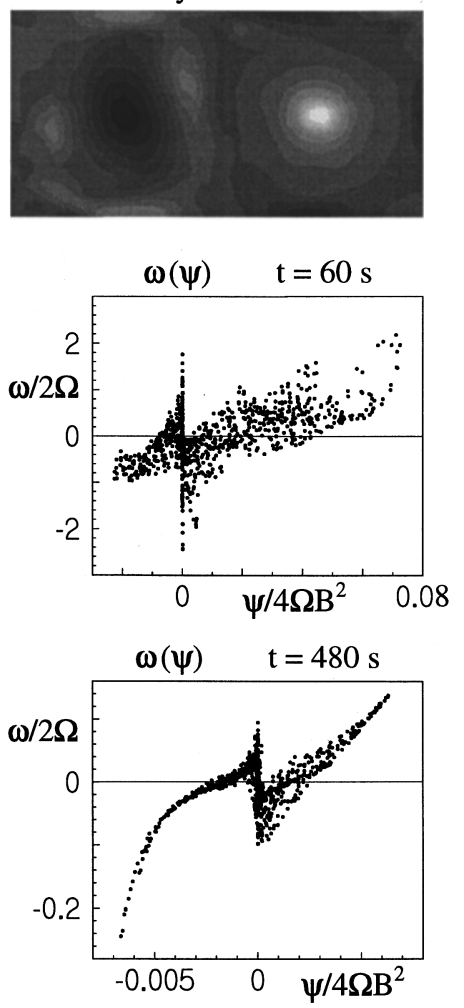

FIG. 2. Experimental results for spin-up from $0 \rightarrow 0.5 \mathrm{rad} / \mathrm{s}$ in geometry no. 1 .

$<y<B$ with a flat bottom, the starting flow is purely horizontal. In that case the continuity equation is given in Cartesian coordinates by

$$
\frac{\partial u}{\partial x}+\frac{\partial v}{\partial y}=0,
$$

so that we can introduce the stream function $\psi$ by

$$
u=\frac{\partial \psi}{\partial y}, \quad v=-\frac{\partial \psi}{\partial x}
$$

Writing the relative vorticity as $-\nabla^{2} \psi$, we find a Poisson equation for the stream function of the starting flow:

$$
\frac{\partial^{2} \psi}{\partial x^{2}}+\frac{\partial^{2} \psi}{\partial y^{2}}=2 \Omega
$$

with $\psi=0$ at $x= \pm L$ and at $y= \pm B$. This problem, though stated in a different form, was solved by Stokes, ${ }^{12}$ and independently by Van Heijst et $a l^{7}{ }^{7}$ The solution is given by 

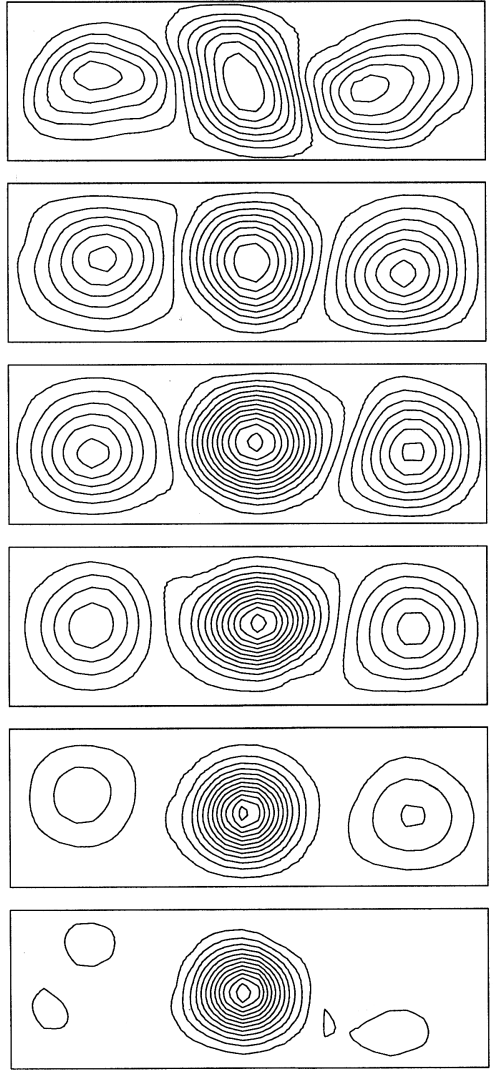

$t=120 \mathrm{~s}$

$t=60 \mathrm{~s}$

$t=30 \mathrm{~s}$$$
t=120 \mathrm{~s}
$$

$t=240 \mathrm{~s}$

$t=480 \mathrm{~s}$
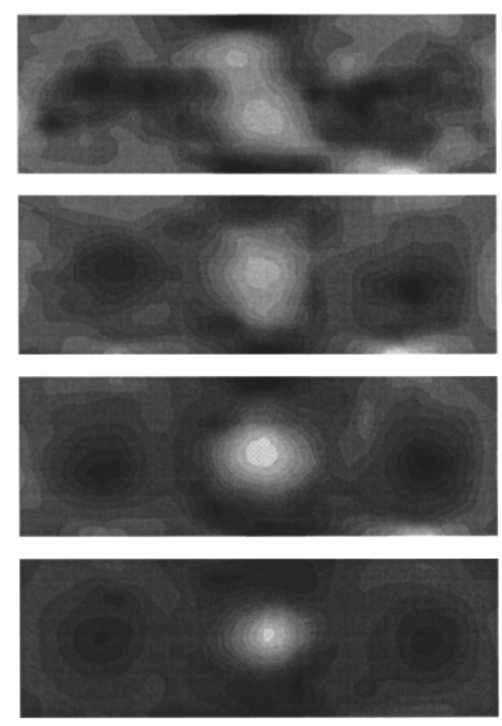

$t=960 \mathrm{~s}$

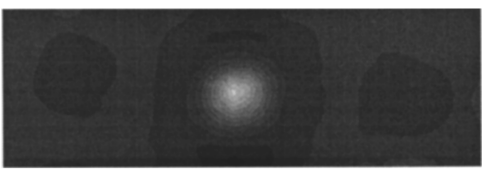

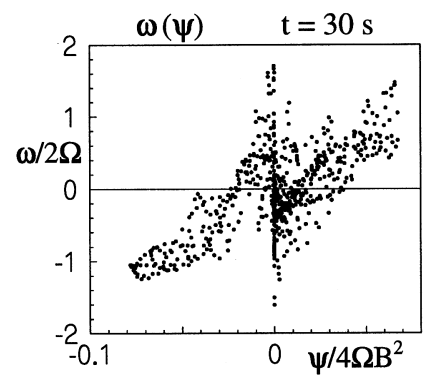
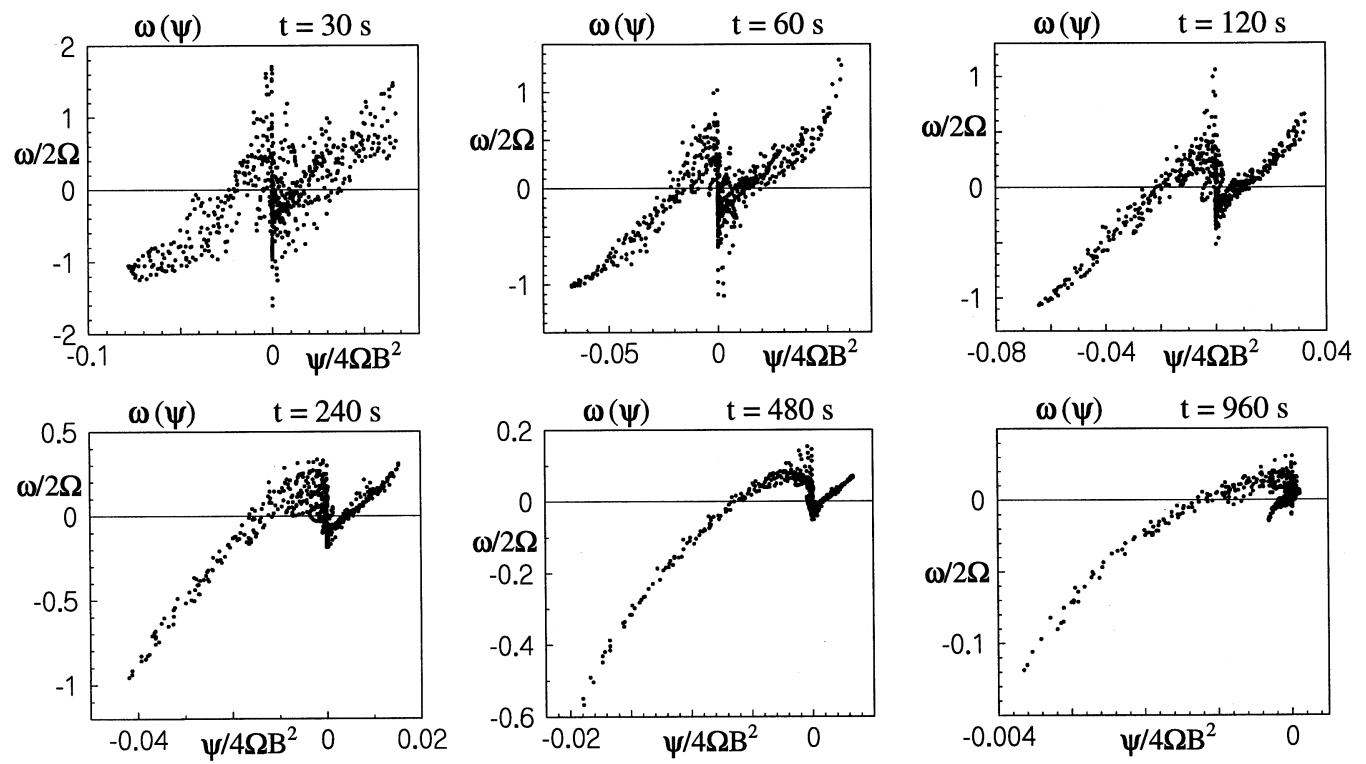

FIG. 3. Experimental results for spin-up from $0 \rightarrow 0.5 \mathrm{rad} / \mathrm{s}$ in geometry no. 2 .

$$
\begin{aligned}
\psi / L^{2} \Omega= & x^{2} / L^{2}-1+4 \sum_{n=0}^{\infty} \frac{(-1)^{n}}{\left(n+\frac{1}{2}\right)^{3} \pi^{3}} \cos \left(n+\frac{1}{2}\right) \pi x / L \\
& \times \frac{\cosh \left(n+\frac{1}{2}\right) \pi y / L}{\cosh \left(n+\frac{1}{2}\right) \pi B / L}
\end{aligned}
$$

where $x^{2} / L^{2}-1$ may be regarded as the particular solution, and the sum as the homogeneous solution of (12).
An explicit expression for the velocity field of the starting flow in a rectangular geometry with a step is much more difficult to find. In the first place, the solution is dependent on the coordinate in the vertical direction, so that the solution can no longer be expressed in terms of a scalar stream function; in the second place, in the case of a step there seem to be no elementary functions that can be used to expand the homogeneous solution of the vorticity equation.

Van de Konijnenberg ${ }^{8}$ gave the solution of a simplified 


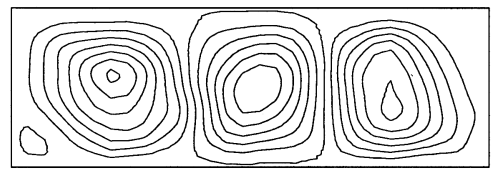

$$
t=30 \mathrm{~s}
$$

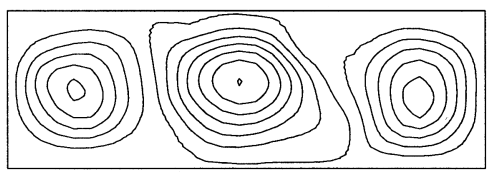

$$
t=60 \mathrm{~s}
$$
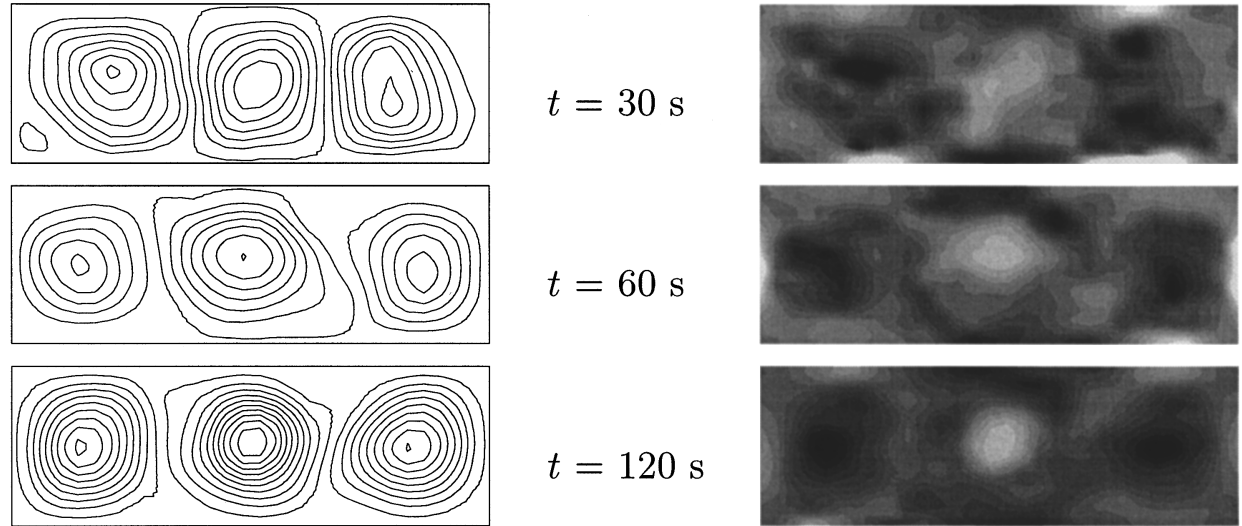

$$
t=120 \mathrm{~s}
$$
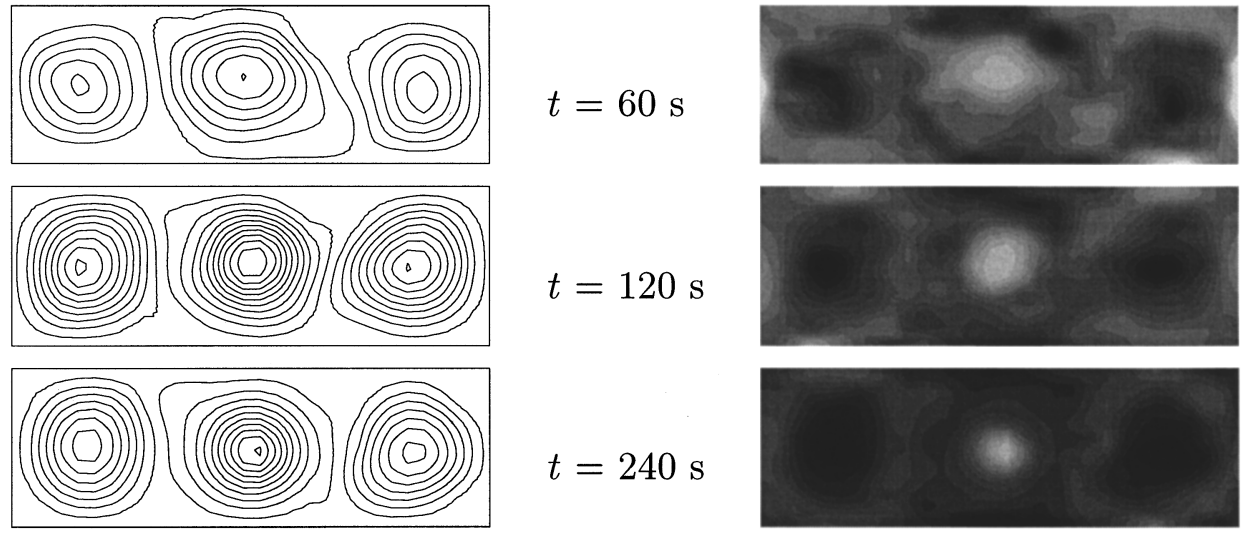

$t=240 \mathrm{~s}$
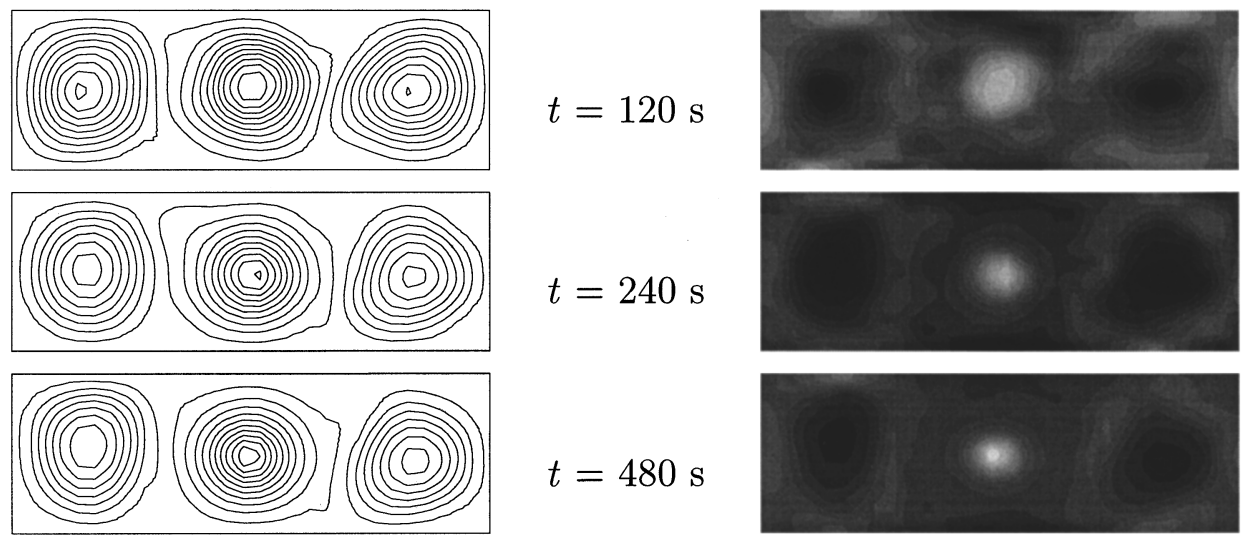

$$
t=480 \mathrm{~s}
$$
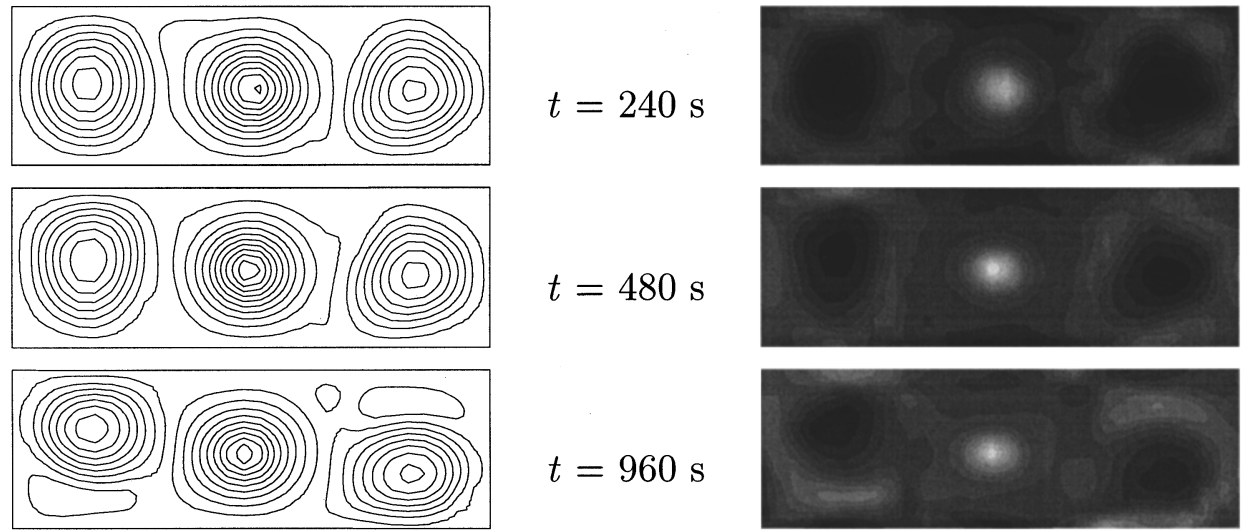

$$
t=960 \mathrm{~s}
$$
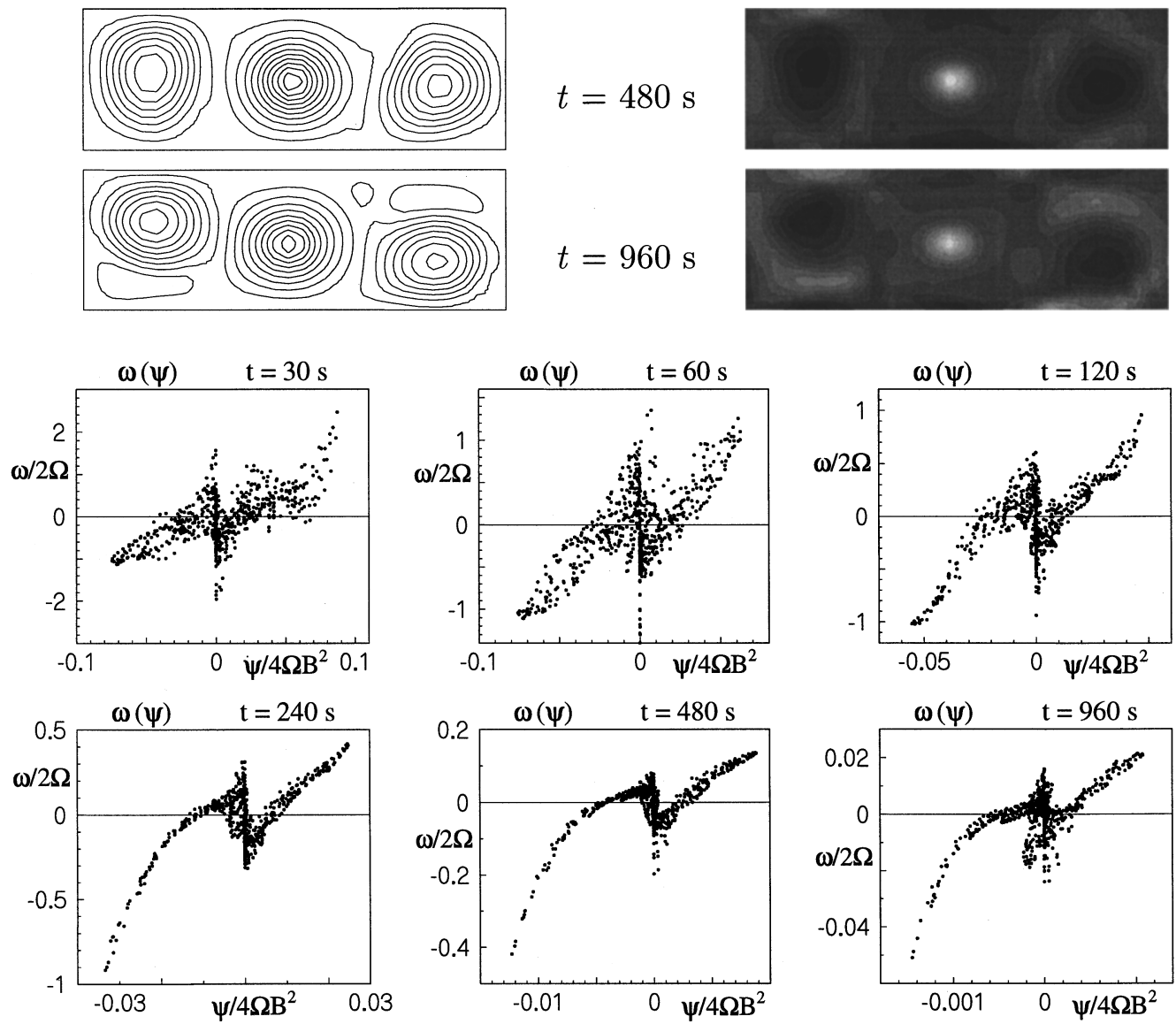

FIG. 4. Experimental results for spin-up from $0 \rightarrow 0.5 \mathrm{rad} / \mathrm{s}$ in geometry no. 3 .

problem, in which the topography is accounted for according to the shallow-water approximation. In this model, the horizontal velocity components $u$ and $v$ are assumed to be independent of $z$. The vertical velocity components depend linearly on $z$, and can be replaced by a divergence in the horizontal plane, so that a problem in two dimensions remains. In the shallow-water approximation, the topography acts as a density function for the two-dimensional flow, so the horizontal velocity field integrated over the local depth $H(x, y)$ is divergence-free:

$$
\frac{\partial}{\partial x}(H u)+\frac{\partial}{\partial y}(H v)=0 .
$$

This leads to a generalized definition of the stream function:

$$
u=\frac{1}{H} \frac{\partial \Psi}{\partial y}, \quad v=-\frac{1}{H} \frac{\partial \Psi}{\partial x}
$$

the capital $\Psi$ is used to indicate the difference between this definition and the definition of the stream function $\psi$ accord- 


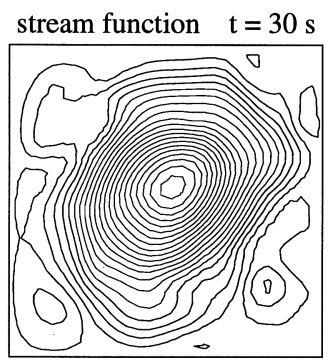

stream function $\mathrm{t}=60 \mathrm{~s}$

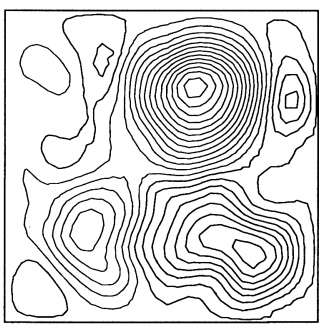

stream function $\mathrm{t}=120 \mathrm{~s}$

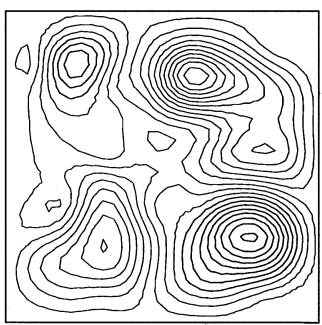

stream function $\mathrm{t}=240 \mathrm{~s}$

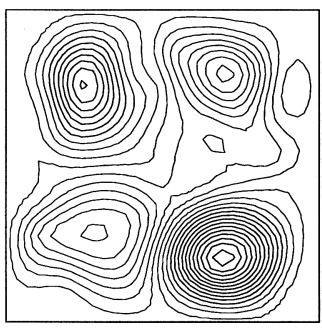

stream function $t=360 \mathrm{~s}$

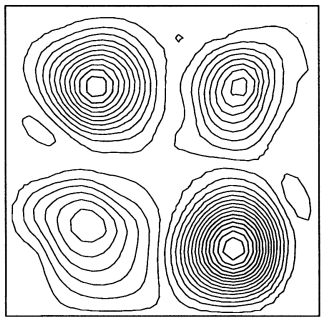

vorticity $t=30 s$

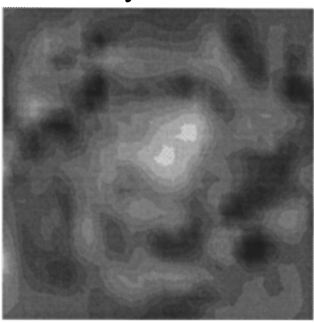

vorticity $t=60 \mathrm{~s}$

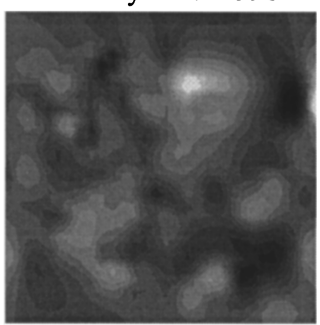

vorticity $t=120 \mathrm{~s}$

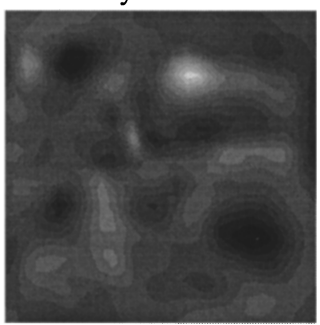

vorticity $t=240 \mathrm{~s}$

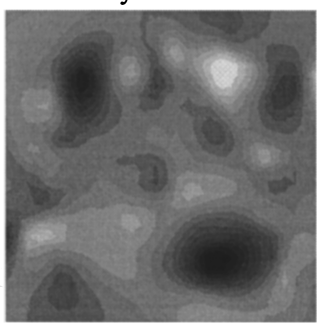

vorticity $t=360 s$

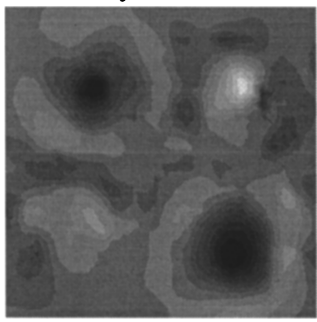

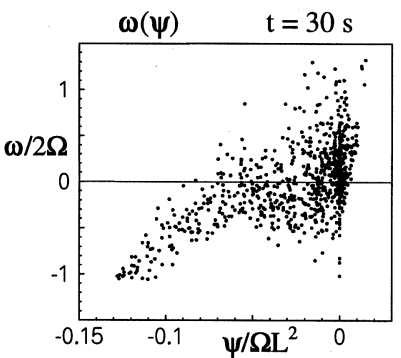
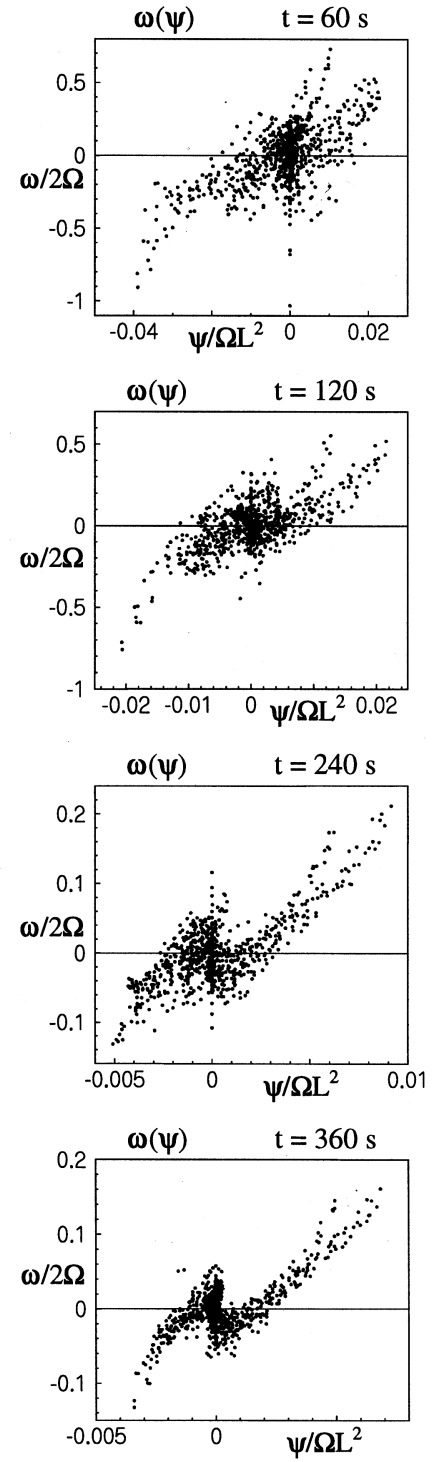

FIG. 5. Dye visualization of spin-up from 0 to $0.5 \mathrm{rad} / \mathrm{s}$ in geometry no. 2 . The figures in the left column correspond to an experiment in which the dye was added at $t=0$, the figures in the right column correspond to an experiment in which the dye was added at $t=180 \mathrm{~s}$. In both experiments the dye was added at the middle of the left sidewall.

ing to (11). Since the relative vorticity has a uniform value $-2 \Omega$, one arrives at an equation for $\Psi$ given by

$$
\frac{\partial^{2} \Psi}{\partial x^{2}}+\frac{\partial^{2} \Psi}{\partial y^{2}}+H \frac{\partial H^{-1}}{\partial x} \frac{\partial \Psi}{\partial x}+H \frac{\partial H^{-1}}{\partial y} \frac{\partial \Psi}{\partial y}=2 \Omega H
$$

Van de Konijnenberg ${ }^{8}$ presented the solution of (16) on a rectangular domain with the depth $H(x)$ given by

$$
\begin{array}{ll}
x<0: & H(x)=H_{0}(1+\xi), \\
x>0: & H(x)=H_{0}(1-\xi),
\end{array}
$$

where $H_{0}$ is the median depth and $\xi$ is a dimensionless measure of the magnitude of the topographic discontinuity at $x=0$. However, this solution should be regarded as an average of the three-dimensional solution over the vertical coordinate $z$, which may be different from the flow observed at 


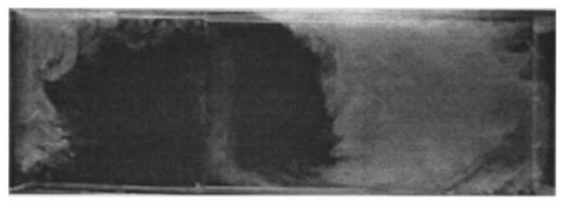

$$
t=25 \mathrm{~s}
$$

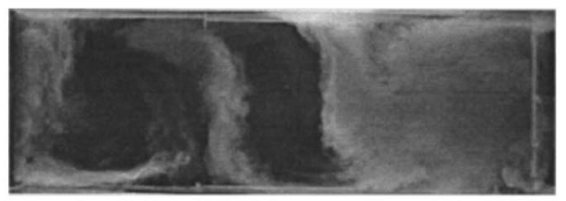

$t=35 \mathrm{~s}$

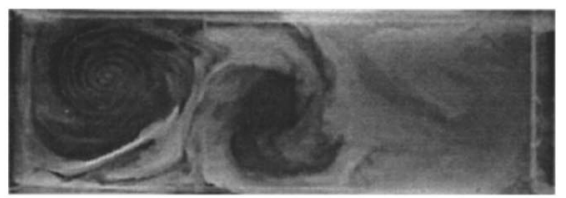

$t=60 \mathrm{~s}$

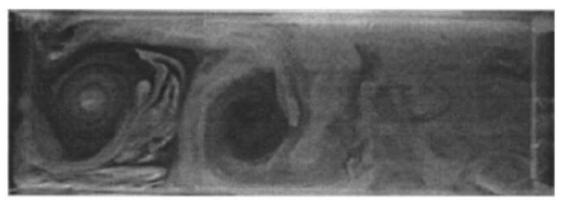

$$
t=120 \mathrm{~s}
$$

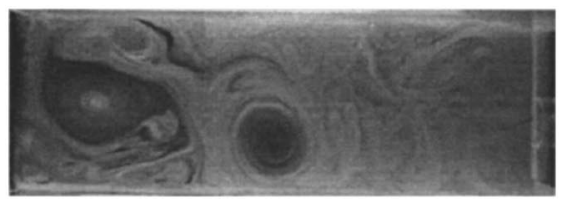

$$
t=210 \mathrm{~s}
$$
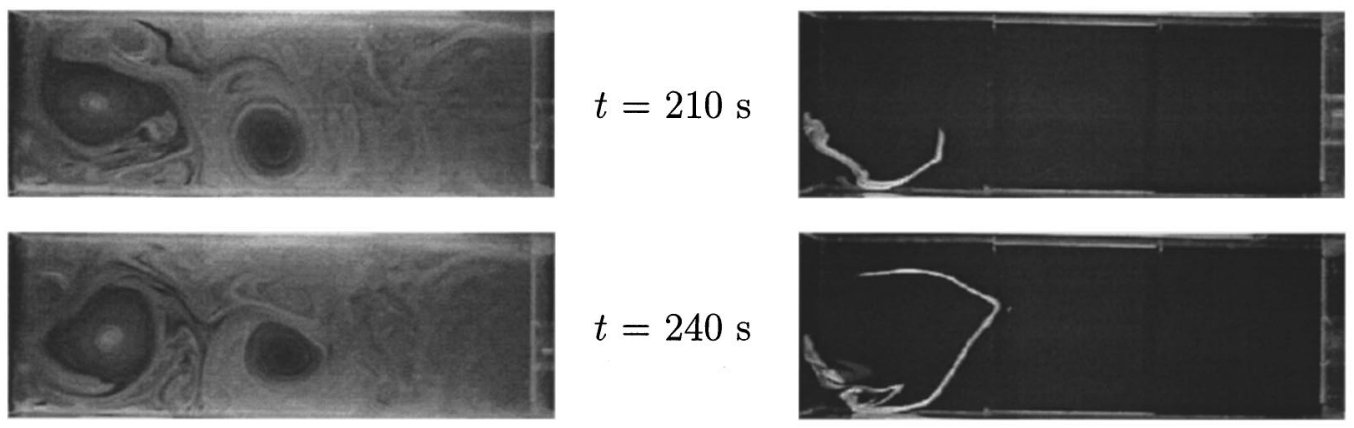

$t=240 \mathrm{~s}$
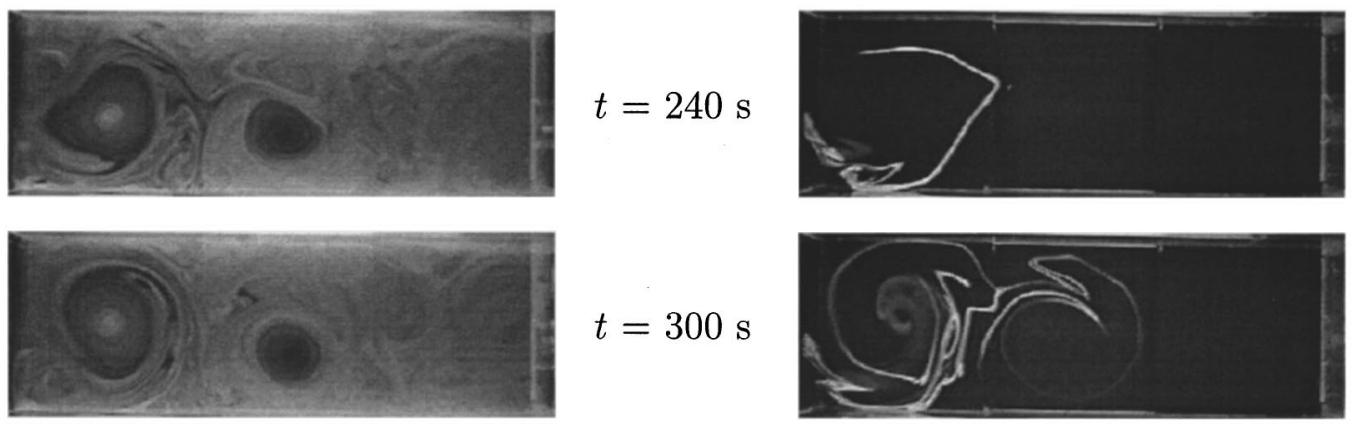

$t=300 \mathrm{~s}$
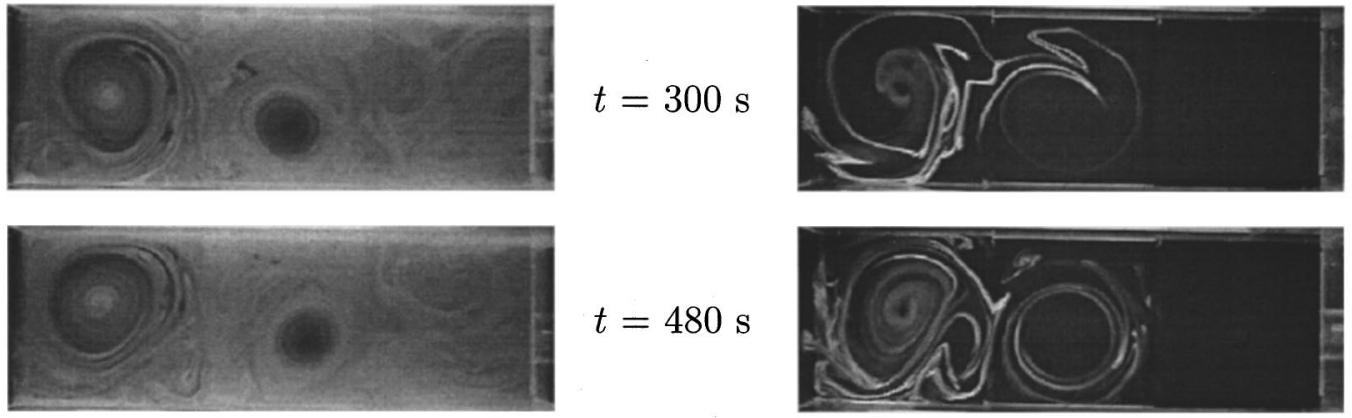

$t=480 \mathrm{~s}$

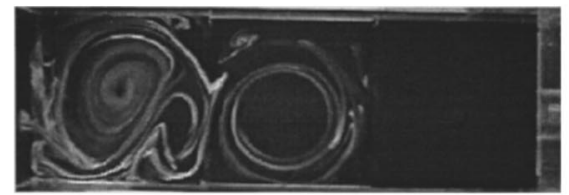

FIG. 6. As in Fig. 5, but for geometry no. 3.

the surface. Further investigations ${ }^{8}$ show that even for a considerable topography height, the flow at the surface shows more resemblance with the solution for a flat bottom than with the solution according to the shallow-water approximation.

\section{EXPERIMENTAL SETUP}

The spin-up experiments were performed by using a tank filled with tap water, placed on a rotating table. At $t=0$, the angular velocity of the table was suddenly increased from 0 to $\Omega$, and was thereafter kept at this value during the experiment. Thus at $t=0$ the Rossby number $U / \Omega L$ is equal to one, but as the relative flow decays in the course of the experiment, the Rossby number gradually decreases to zero. In all experiments, the final angular velocity $\Omega$ was chosen to be $0.5 \mathrm{rad} / \mathrm{s}$. It was verified that fluctuations in the final angular velocity are negligible compared with the final angular velocity used in the experiments. Four configurations were used, each consisting of one or two square subdomains with a uniform depth of $20 \mathrm{~cm}$, and one or two square subdomains with a uniform depth of $15 \mathrm{~cm}$. These configurations are shown in Fig. 1.

Quantitative results were obtained with small tracer particles floating at the surface of the fluid. Dye was added to the water in order to increase the contrast between the fluid and the particles. First, a video recording of the flow was made with a video camera corotating with the tank. Then, after the experiment, the recording was processed by a PC 


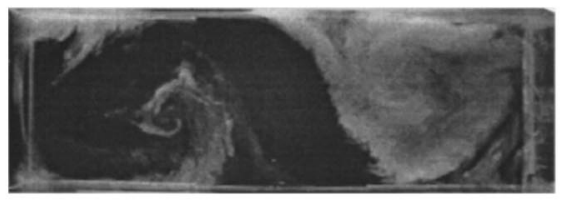

$t=25 \mathrm{~s}$

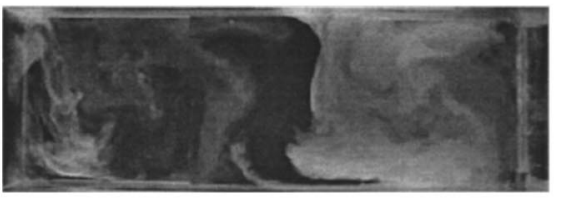

$t=35 \mathrm{~s}$

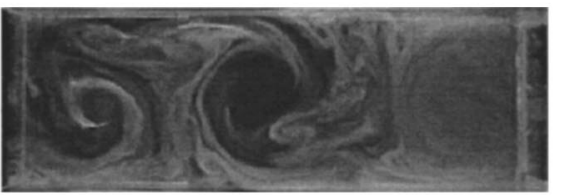

$t=60 \mathrm{~s}$

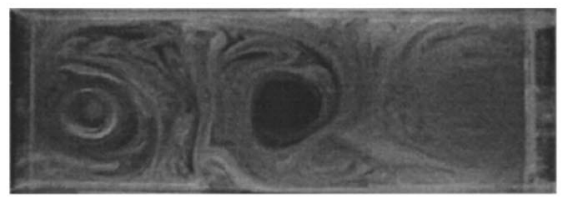

$t=120 \mathrm{~s}$

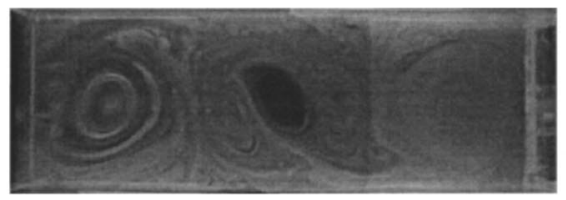

$t=210 \mathrm{~s}$
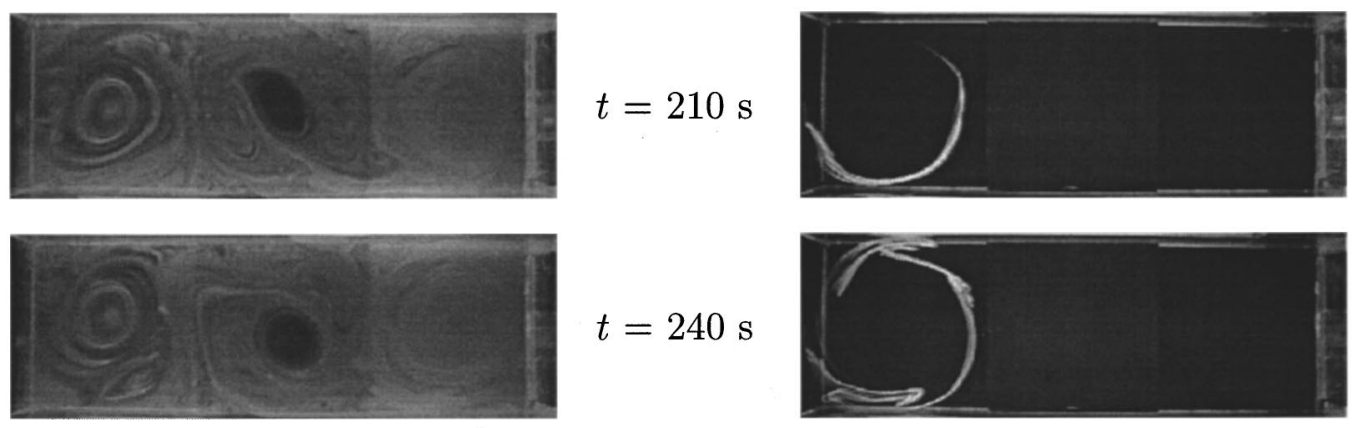

$t=240 \mathrm{~s}$
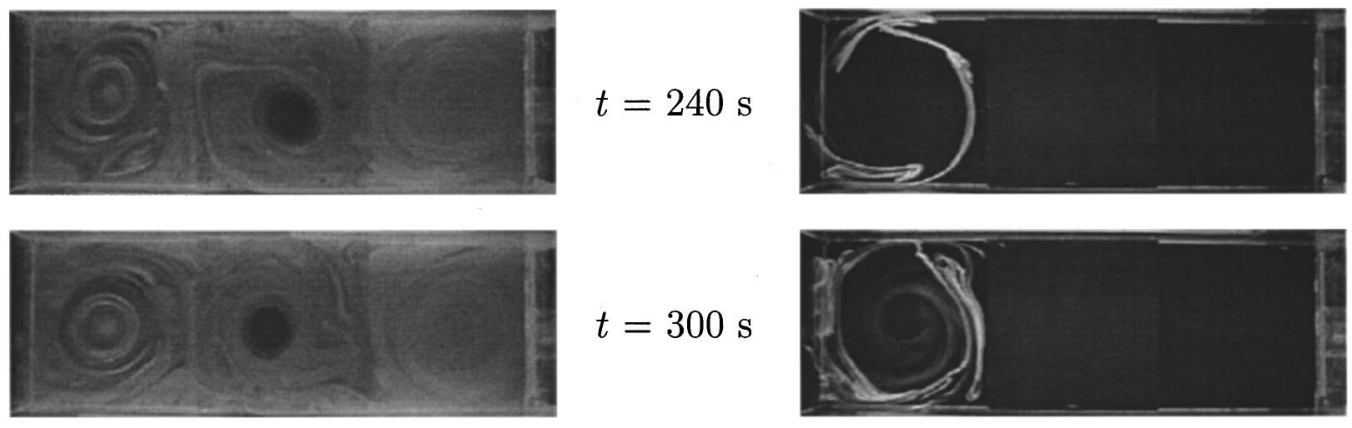

$t=300 \mathrm{~s}$
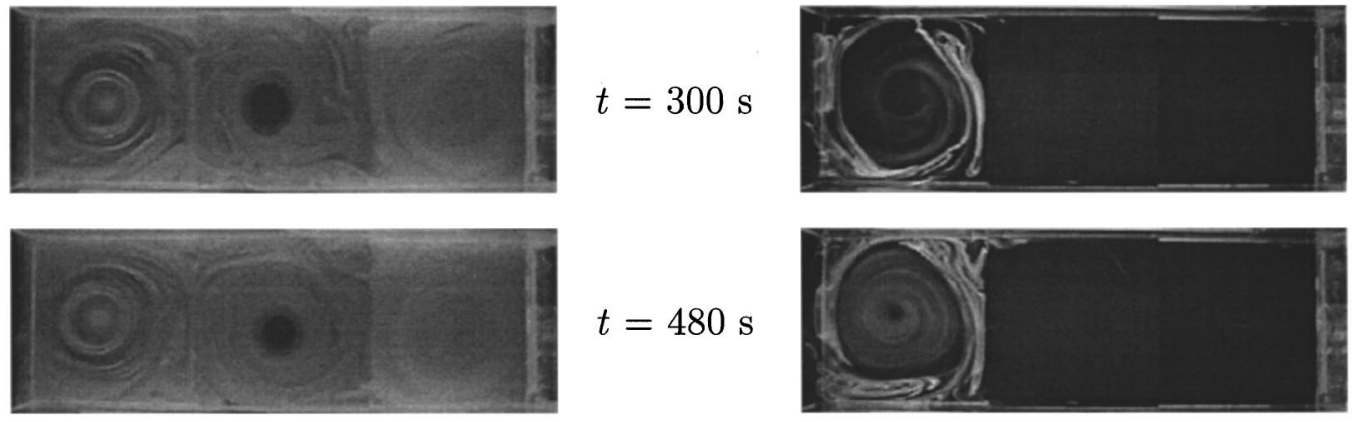

$t=480 \mathrm{~s}$

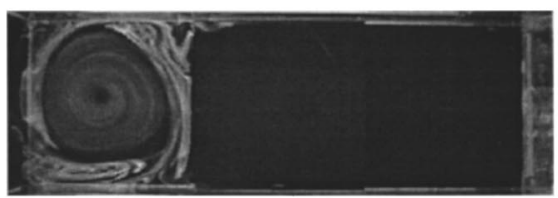

FIG. 7. Experimental results for spin-up from $0 \rightarrow 0.5 \mathrm{rad} / \mathrm{s}$ in geometry no. 4 .

equipped with a frame grabber. For this purpose, an adapted version of the DigImage system developed by Dalziel ${ }^{13}$ was used. This is an image processing system that allows the tracking of particles based on a number of user-defined criteria such as brightness and size. Further processing of the particle trajectory data was made possible by an extension to the DigImage software developed by Van der Plas. ${ }^{14}$ This option provides the possibility to extract data files containing the particle velocities from the data file created by the particle tracking routine. The vorticity was obtained by fitting the data with spline functions and manipulating the coefficients of this expansion. The stream function was calculated from the vorticity by using a Poisson solver. In this way the stream function of the solenoidal component of the velocity field is calculated, which is more elegant than applying integration techniques if the flow is not exactly divergence free.

\section{EXPERIMENTAL RESULTS}

\section{A. Geometry no. 1}

The results of this experiment are presented in Fig. 2. The data have been represented using the stream function defined by (11), that is, the quantity that is presented in the graphs is the solution of $\nabla^{2} \psi=-\omega$, not of $\nabla^{2} \Psi=-\nabla \times H \mathbf{u}$. In view of the tenet that streamlines should run parallel to the 
velocity field, this approach leads to better results. Apparently, for the flow at the free surface, $\nabla \cdot \mathbf{u}=0$ is a better assumption than $\nabla \cdot(H \mathbf{u})=0$.

Both the experimentally observed starting flow at the free surface and the formation of vortices in the corners are similar to corresponding experiments with a flat bottom. ${ }^{7,8}$ However, both a comparison of quantitative measurements and dye visualizations indicate that the flow is now more turbulent, a result of flow separation from the topography. As in similar experiments with a flat bottom, the cyclonic vortices merge into a single cell in the center of the tank at an early stage. However, rather than remaining above the step, the resulting cyclonic vortex moves to the deeper part of the tank. The flow eventually relaxes to a pattern with two cells, separated by the discontinuity in the topography, in qualitative agreement with the restriction of zero cross flow over the topography contours imposed by the Taylor-Proudman theorem. In the beginning of the experiment, the flow is neither stationary nor linear, but the experiments show that the reluctance to cross the step becomes manifest in an early stage, dividing the flow into separate regions long before the relative velocities have become small.

The graphs of $\omega$ versus $\psi$ provide a measure of the instationarity of the flow, and represent the structure of each vortex cell. According to the decreasing amount of scatter in the $\omega(\psi)$ graphs at 120,240 , and $480 \mathrm{~s}$, the flow becomes organized into an increasingly stationary pattern. The curvature of the branches is determined by the formation process of the cells, and by a gradual evolution through Ekman pumping. Because of the nonlinearity of the Ekman pumping term in (7), this evolution is asymmetric with respect to the cyclonic vortex and the anticyclonic vortex.

\section{B. Geometry nos. 2 and 3}

The results for geometry nos. 2 and 3, represented in Figs. 3 and 4, appear to be comparable. In both experiments, vortices are formed in the corners of the domain. However, in contrast with the corresponding experiment with a flat bottom, merging does not occur. Apparently the corner vortices cannot cross the step, and remain separated by the middle square region. Thus the resulting cycloneanticyclone-cyclone arrangement is determined in an early stage of the experiment, and any possible tendency of the cyclonic vortices to move under the influence of the sidewalls or a deformed free surface does not find expression.

The nonuniform depth leads to different spin-up time scales for the individual square regions. Application of (9) leads to $\tau=212 \mathrm{~s}$ for the shallower parts and $\tau=283 \mathrm{~s}$ for the deeper parts. In the linear case, this would result in the vortices in the deeper parts being about three times as strong as the vortices in the shallower parts after $960 \mathrm{~s}$. The sizes of the branches in the $\omega(\psi)$ graphs at $960 \mathrm{~s}$ indicate that in reality, the cyclonic vortices are much weaker than this estimate. This is probably caused by the nonlinearity in the vortex stretching term in (7), and by the cyclonic vortices being bounded by solid walls at more sides. It can be shown ${ }^{8}$ that $960 \mathrm{~s}$ is about the time scale on which the viscous effects begin to become important, so that in particular the cyclonic cells are damped more strongly than by Ekman pumping only.

Of the experiments in geometry nos. 2 and 3 dye visualizations have been made; the results are presented in Figs. 5 and 6. Although in neither experiment merging occurs, considerable mixing of the fluid takes place; the dye is spread over the whole domain with the exception of the cores of the anticyclonic cell and the left cyclonic cell. In particular in geometry no. 3 the dye is confined to a square region bounded by the topography discontinuity. Since the vortex in this square is cyclonic, the motion in the Ekman layer is directed inward, so that in a top view of the dye distribution, the whole vortex seems to be dyed.

The figures of the experiment in geometry no. 2 show a different behavior. If dye is added at $t=0$ the appearance of the dye distribution looks similar to the corresponding experiment in geometry no. 3 , but if the dye is added at $180 \mathrm{~s}$, a "leak" from the left cyclonic cell to the central anticyclonic cell becomes apparent. Further experiments with dye have shown that this leak is caused by a weak flow across the step toward the central cell, compensated by a flow in the reverse direction in the Ekman layer. At this moment it is not clear why this cross-flow occurs in geometry no. 2 and not in geometry no. 3 . The effect might be connected with a difference in strength between the cyclonic and anticyclonic vortices, which is only present in geometry no. 2 .

\section{Geometry no. 4}

The results for the experiment in geometry no. 4 are given in Fig. 7. As in geometry nos. 1, 2, and 3 the starting flow has the same appearance as in a tank without a topography, but separation from the topography immediately takes place, and the flow becomes turbulent. The vorticity graphs at $30 \mathrm{~s}$ show that the turbulence affects the flow in the whole domain. Nevertheless, the central anticyclonic vortex remains identifiable. Between $t=30 \mathrm{~s}$ and $t=60 \mathrm{~s}$ it moves to one of the shallow parts, and remains there until the fluid has spun up. In the rest of the domain new vortices are formed, so that a streamline pattern emerges with opposite circulation in two adjoining quadrants. This situation is in striking contrast with experiments in a square tank with a flat bottom, ${ }^{8}$ where the flow keeps the appearance of a single cell. As in geometry no. 1, the topography affects the streamline pattern at the surface in an early stage, but even after $360 \mathrm{~s}$ the vorticity profiles are not really smooth. Nevertheless, this experiment provides a clear example of topographic forcing of the flow into a certain cellular pattern.

\section{DISCUSSION}

Out of the four geometries discussed in this chapter, the topography is symmetry-breaking in geometry nos. 1 and 4 only. In those experiments, cyclonic vortices end up in the deeper part and anticyclonic vortices in the shallower part. According to conservation of potential vorticity, this is what is to be expected: Some vortex tubes descend the step and are stretched, others climb the step and are squeezed, so fluid in the deeper parts will acquire cyclonic vorticity, and fluid in the shallower parts will acquire anticyclonic vorticity. 
Additional experiments indicate that the extent to which topographic forcing of a particular vortex pattern occurs depends on the relative height of the topography. If the topography is very weak, its influence on the flow at the surface becomes noticeable only if the relative velocities have become very small. If on the other hand the height of the topography is large, the spin-up time scale in the shallow parts becomes much shorter than in the deep parts, resulting in strong asymmetry between the cyclonic and anticyclonic cells. This difference is already noticeable in the experiments discussed in this paper.

In geometry no. 4 the separation into cyclonic and anticyclonic regions is particularly clear. However, by the time the organization is more or less complete, the flow has lost much of its initial energy. It is therefore doubtful whether a similar lattice of counterrotating cyclonic and anticyclonic vortices would appear in a geometry with more than four topographic subdomains.

\section{ACKNOWLEDGMENT}

One of us (J.v.d.K.) gratefully acknowledges financial support from the Dutch Foundation of Fundamental Research (FOM).
${ }^{1}$ B. M. Boubnov, S. B. Dalziel, and P. F. Linden, "Source-sink turbulence in a stratified fluid,' J. Fluid Mech. 261, 273 (1994).

${ }^{2}$ J. C. McWilliams, "The emergence of isolated coherent vortices in turbulent flow,' J. Fluid Mech. 146, 21 (1984).

${ }^{3}$ B. Legras, P. Santangelo, and R. Benzi, "High-resolution numerical experiments for forced two-dimensional turbulence," Europhys. Lett. 5(1), 37 (1988).

${ }^{4}$ H. P. Greenspan and L. N. Howard, "On a time-dependent motion in a rotating fluid,"' J. Fluid Mech. 17, 385 (1963).

${ }^{5} \mathrm{E}$. H. Wedemeyer, "The unsteady flow within a spinning cylinder," J. Fluid Mech. 20, 383 (1964).

${ }^{6}$ G. J. F. van Heijst, "Spin-up phenomena in non-axisymmetric containers," J. Fluid Mech. 206, 171 (1989).

${ }^{7}$ G. J. F. van Heijst, P. A. Davies, and R. G. Davis, "Spin-up in a rectangular container," Phys. Fluids A 2, 150 (1990).

${ }^{8}$ J. A. van de Konijnenberg, "Spin-up in non-axisymmetric containers," $\mathrm{Ph} . \mathrm{D}$. thesis, Eindhoven University of Technology, The Netherlands, 1995.

${ }^{9}$ G. J. F. van Heijst, L. R. M. Maas, and C. W. M. Williams, "The spin-up of fluid in a rectangular container with a sloping bottom,' J. Fluid Mech. 265, 125 (1994).

${ }^{10}$ H. P. Greenspan, The Theory of Rotating Fluids (Cambridge U. P., Cambridge, England, 1968).

${ }^{11}$ J. Pedlosky, Geophysical Fluid Dynamics (Springer-Verlag, Berlin, 1987).

${ }^{12}$ G. G. Stokes, "On some cases of fluid motion," Trans. Cambridge Philos. Soc. VIII (1843), reprinted in Mathematical and Physical Papers (Cambridge U. P. Cambridge, England, 1880), Vol. I.

${ }^{13}$ S. Dalziel, “DigImage. Image processing for fluid dynamics," Cambridge Environmental Research Consultants Ltd., 1992.

${ }^{14}$ G. A. J. van der Plas, "Introduction manual for particle tracking with DigImage," Internal report R-1323-D, Faculty of Technical Physics, Fluid Dynamics Laboratory, Eindhoven University of Technology, The Netherlands, 1994. 